\title{
Decay of an ultracold fermionic lithium gas near a Feshbach resonance
}

\author{
K. Dieckmann, C. A. Stan, S. Gupta, Z. Hadzibabic, C. H. Schunck, and W. Ketterle \\ Department of Physics, MIT-Harvard Center for Ultracold Atoms, and Research Laboratory of Electronics, \\ MIT, Cambridge, MA 02139
}

(November 5, 2018)

\begin{abstract}
We studied the magnetic field dependence of the inelastic decay of an ultracold, optically trapped fermionic ${ }^{6} \mathrm{Li}$ gas of different spin compositions. The spin mixture of the two lowest hyperfine states showed two decay resonances at $550 \mathrm{G}$ and $680 \mathrm{G}$, consistent with the predicted Feshbach resonances for elastic s-wave collisions. The observed lifetimes of several hundred milliseconds are much longer than the expected time for Cooper pair formation and the phase transition to superfluidity in the vicinity of the Feshbach resonance.
\end{abstract}

PACS numbers: 34.50.-s, 05.30.Fk,39.25.+k, 32.80.Pj

Interactions between atoms can be strongly modified by tuning magnetic fields to Feshbach resonances where a molecular state has the same energy as the colliding atoms. This mechanism has been used to dramatically alter the properties of ultracold bosonic gases [1-4]. For degenerate Fermi gases, such control over the interaction strength is crucial in the search for a superfluid phase transition. For dilute Fermi gases, the predicted phase transition occurs at temperatures that are experimentally not accessible [5], unless the scattering length is resonantly enhanced. In this case, as was pointed out by $[6-10]$, the transition temperature can be comparable to the temperatures achieved in current experiments [11-16].

Promising candidates for an experimental observation of fermionic superfluidity are ${ }^{6} \mathrm{Li}$ and ${ }^{40} \mathrm{~K}$. For an optically trapped mixture of two spin states of ${ }^{40} \mathrm{~K}$, a Feshbach resonance has been observed by measuring the thermalization time of the gas [17]. For an optically trapped spin mixture of the two lowest Zeeman states of ${ }^{6} \mathrm{Li}$, a wide s-wave Feshbach resonance has been predicted first by [18]. Experiments with ${ }^{6} \mathrm{Li}$ have so far only observed a magnetic field dependence of the elastic cross section far away from the predicted resonance [14]. Near Feshbach resonances, the enhancement of the scattering length is usually accompanied by enhanced inelastic collisions which lead to rapid trap loss. This signature was used to identify Feshbach resonances in bosonic gases [20-22]. However, inelastic losses have also posed a severe limitation for experiments near Feshbach resonances, in particular at high atomic densities. The superfluid phase transition for fermions will only be observable if the time for the formation of Cooper pairs is shorter than the decay time of the gas. For fermions, inelastic decay in the s-wave channel can be suppressed due to the Pauli exclusion principle. However, even in the zero-temperature limit the kinetic energy of the cloud is of the order of the Fermi energy. Therefore, inelastic collisions for higher partial waves are expected to limit the lifetime of the gas.
This letter is the first report on the study of inelastic collisions in a fermionic system near Feshbach resonances. We have observed resonant magnetic field dependent inelastic decay of an ultracold, optically trapped spin mixture of ${ }^{6} \mathrm{Li}$.

The ultracold lithium samples were prepared by sympathetic cooling of ${ }^{6} \mathrm{Li}$ by ${ }^{23} \mathrm{Na}$ as described previously [15]. The remaining ${ }^{23} \mathrm{Na}$ atoms were removed from the magnetic trap by rf induced spin-flips to untrapped states. This typically produced $3 \times 10^{5}$ lithium atoms in the $|1 / 2,-1 / 2\rangle$ state at a temperature of $400 \mathrm{nK}$, equal to half the Fermi temperature. The atoms were transferred into an optical trap formed by a single far detuned beam with up to $1 \mathrm{~W}$ of power at $1064 \mathrm{~nm}$. The beam had a $14 \mu \mathrm{m}$ waist and was aligned horizontally along the symmetry axis of the magnetic trap. This generated a $175 \mu \mathrm{K}$ deep trapping potential, with $12 \mathrm{kHz}$ radial and $200 \mathrm{~Hz}$ axial trapping frequencies. Prior to the transfer, the cloud was adiabatically decompressed in the radial direction during $1 \mathrm{~s}$ to improve the spatial overlap with the optical trap. After this stage, the trap frequencies in the magnetic trap were $149 \mathrm{~Hz}$ radially and $26 \mathrm{~Hz}$ axially. We then adiabatically ramped up the power of the optical trap over $500 \mathrm{~ms}$. Subsequently, the magnetic trapping fields were ramped down in $100 \mathrm{~ms}$, leaving a $1.5 \mathrm{G}$ guiding field along the trap axis. After the transfer, the cloud contained $3 \times 10^{5}$ atoms at $3 \times 10^{13} \mathrm{~cm}^{-3}$ peak density and $22 \mu \mathrm{K}$ temperature, close to the $21 \mu \mathrm{K}$ Fermi temperature. We attribute the rise in temperature relative to the Fermi temperature to residual excitations during the transfer into the optical trap. (We often observed axial oscillations of the cloud after the transfer.)

We studied inelastic decay for three different spin compositions of the cloud. The lithium atoms were either trapped purely in the lowest $(|1\rangle)$, or the second to lowest $(|2\rangle)$ energy state, or in a $50 \%-50 \%$ mixture of these two Zeeman states. At low magnetic fields, the states $|1\rangle$ and $|2\rangle$ correspond to the $\left|F, m_{F}\right\rangle=|1 / 2,+1 / 2\rangle$ and $|1 / 2,-1 / 2\rangle$ states, respectively. A full transfer $|1 / 2,-1 / 2\rangle \rightarrow|1 / 2,+1 / 2\rangle$ was done at low magnetic field 
by applying a $1 \mathrm{~s}$ rf-driven adiabatic passage between the two states, which was $>95 \%$ complete. The spin mixture was produced by a faster, non-adiabatic rf-sweep of $200 \mathrm{~ms}$ duration. The population of the states was analyzed by applying a $7 \mathrm{G} / \mathrm{cm}$ magnetic field gradient along the trap axis with a $6.5 \mathrm{G}$ offset field in the center, and reducing the strength of the optical confinement. This resulted in full spatial separation of the two spin states in the optical trap. Resonant absorption imaging was used to determine the atom number in each of the spin states. Using a full transfer we compared the absorption cross sections for circularly polarized light for the two spin states and found a ratio of $1: 1.2$. Taking this into consideration, we were able to control the relative population of the spin states by rf-sweeps with an accuracy of $\pm 4 \%$.

In order to study the decay of the cloud near the Feshbach resonance, predicted to occur at about $800 \mathrm{G}$ [18], we applied magnetic fields up to $900 \mathrm{G}$ using the anti-bias coils of the cloverleaf magnetic trap [23]. The magnetic field strength was calibrated in two independent ways to $2 \%$ accuracy. For calibration of magnetic fields up to $100 \mathrm{G}$, we loaded ${ }^{23} \mathrm{Na}$ into the optical trap and drove rf transitions to magnetically untrapped states. Resonances were observed by measuring the remaining atom number after recapture into the magnetic trap. As a second method at about $700 \mathrm{G}$, we used direct absorption imaging of ${ }^{6} \mathrm{Li}$ in the optical trap in the presence of higher magnetic fields. The magnetic field values were then derived from the frequency shifts of the observed resonances from the lithium D2 line. We also verified that drifts of the magnetic field during the pulses, occurring from thermal expansion of the coils due to the high current load, were negligible.

We measured the magnetic field dependence of the decay by measuring the atom number at two different times, $50 \mathrm{~ms}$ and $500 \mathrm{~ms}$ after switching on the magnetic field within about $4 \mathrm{~ms}$. For measuring the remaining atom number, the magnetic field was rapidly switched off within $100 \mu \mathrm{s}$, and the cloud was probed by absorption imaging at low magnetic field. Normalizing the number at long time to the number at short time made the measurement less sensitive to atom number drifts and initial losses from the optical trap. These losses can occur due to the sloshing motion of the cloud and due to initial evaporation.

For the cloud purely in state $|2\rangle$, we observed no significant decay over the entire range of magnetic fields, as can be seen in Figure 1, a). This also confirmed that during the measurement interval, one-body decay (e.g. due to collisions with particles from the background gas) was negligible.

The surviving fraction of the mixture is shown in Figure $1, \mathrm{c})$. No significant decay was observed at low magnetic fields. At higher magnetic field, we found two decay resonances. A strong resonance occurred at $680 \mathrm{G}$ with considerable losses over a range of approximately $100 \mathrm{G}$. At even higher magnetic fields, the decay persisted at a weaker but constant level. In a more detailed scan, shown in Figure 1, d), a second, much weaker and narrower resonance was found at $550 \mathrm{G}$, with an

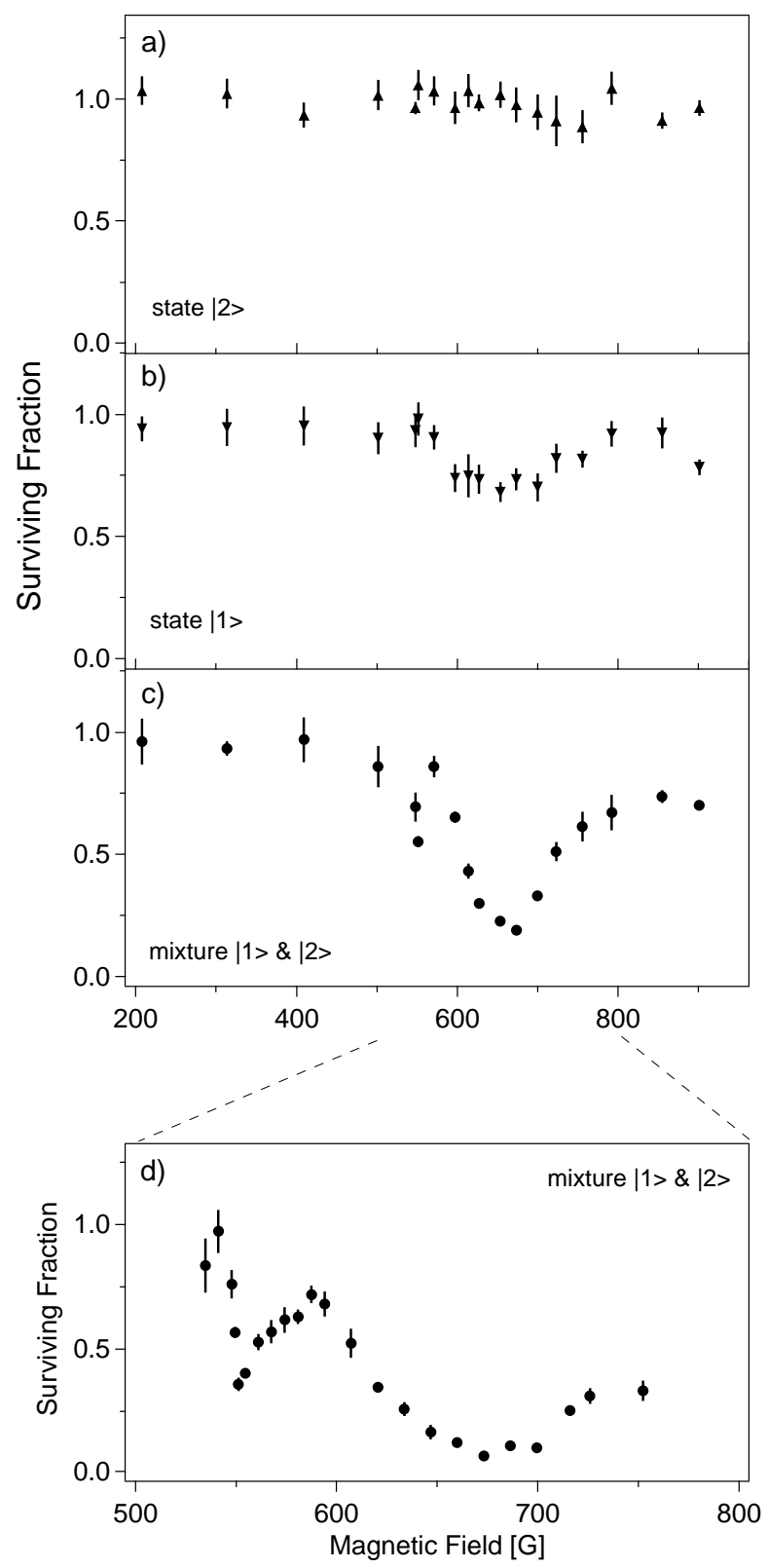

FIG. 1. Magnetic field dependence of inelastic decay in clouds of fermionic ${ }^{6} \mathrm{Li}$. The fraction of atoms remaining after the $500 \mathrm{~ms}$ magnetic field pulse is shown for different spin compositions of the cloud. a) For the state $|2\rangle$, no significant loss was observed. b) The energetically lowest state $|1\rangle$ exhibits a weak decay resonance at $\approx 680 \mathrm{G}$. c) The $50 \%-50 \%$ mixture of two spin states shows two decay resonances, at $550 \mathrm{G}$ and 680 G. d) The two resonances are shown with higher density of data points and for $2 \mathrm{~s}$ magnetic field pulses. Each data point represents an average of three measurements. 
approximate width of $20 \mathrm{G}$. The weaker resonance became more pronounced after $2 \mathrm{~s}$ of dwell time in the magnetic field, whereas the stronger resonance showed "saturation" broadening.

We also measured the time evolution of the atom number at the two resonances. For a two-body (three-body) process the loss rate of atoms $\dot{N}$ is proportional to $N^{2}$ $\left(N^{3}\right)$, where $\mathrm{N}$ is the number of trapped atoms. The decay curves at $680 \mathrm{G}$ are shown in Figure 2. At both resonances we found that the values for $1 / N$ showed a linear dependence on time, characteristic for a two-body process. In order to test for three-body decay we plotted the same data as $1 / N^{2}$. The nonlinear behaviour is not compatible with a simple three-body decay process.

Another experimental observation is the almost complete disappearance of the mixed cloud in Figure 1, d). A resonantly enhanced three-body process would involve two atoms of opposite spin colliding, and a third in either of the spin states. Starting with a $50 \%-50 \%$ mixture, the decay would stop when all atoms in state $|1\rangle$ (or in state $|2\rangle)$ are used up. Therefore, three-body decay can only be consistent with the observation of complete disappearance, if the decay rate does not depend on the spin state of the third particle. In case of strongly different rates for the two spin orientations, the surviving fraction could not drop below $25 \%$.

With the observation of two resonances and the position of the strongest decay of the main resonance deviating from the theoretical prediction [18], the question arises whether the observed decay of the spin mixture can be interpreted as a signature of the Feshbach resonance for elastic s-wave collisions. After the submission of this paper new improved theoretical calculations exhibited a second narrow Feshbach resonance for elastic collisions in the s-wave channel at $550 \mathrm{G}$ [19], in good agreement with the position of the narrow decay resonance. The predicted magnetic field for the main resonance is $860 \mathrm{G}$. However, due to the huge width of the resonance it seems possible that the decay observed at $680 \mathrm{G}$ is related to this s-wave resonance.

The measured decay curves suggest a two-body type of decay. Due to the Pauli exclusion principle dipolar relaxation is not possible in the s-wave channel [24]. Dipolar relaxation in the p-wave channel is possible, as even in the zero-temperature limit the kinetic energy of the cloud is of the order of the Fermi energy, and collisions in the p-wave channel do not completely freeze out. However, no occurrences of resonances in the dipolar decay are theoretically predicted [25].

Therefore, it is most likely that the observed decay is a signature of the Feshbach resonances for the elastic collisions, resulting in enhanced three-body decay. At present no exact theoretical description for the three-body decay mechanism of fermions near a Feshbach resonance is available. Three-body decay is not supported by the measured decay curves. However, one possibility is that

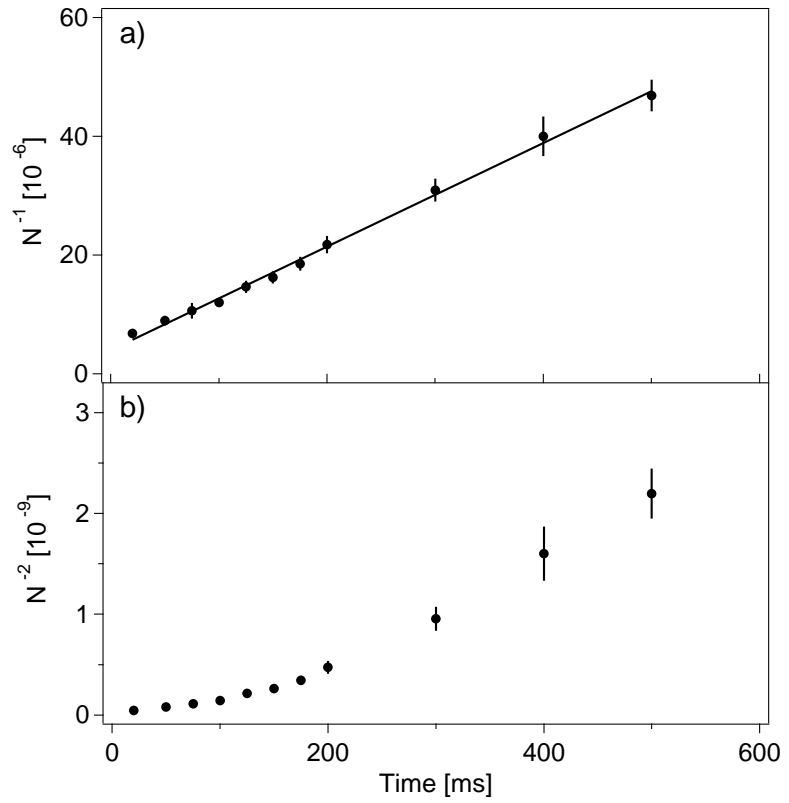

FIG. 2. Decay of the atom number at $680 \mathrm{G}$. a) The data plotted as $1 / N$ show a linear time dependency, consistent with two-body decay. b) The same data plotted as $1 / N^{2}$ clearly show non-linear dependency. For the resonance at $550 \mathrm{G}$, the comparison of least square fits also revealed consistency with a two-body decay.

the decay curve is affected by a change in temperature. An accurate measurement of the temperature was difficult due to technical reasons and a low signal to noise ratio, as the absorption signal drops significantly during the decay. If the sample had cooled down during the decay (e.g. due to an energy dependence of the loss rate) it could speed up the decay in a way that three-body loss results in a decay curve similar to a curve for twobody losses at constant temperature. Another possibility for the deviation from a three-body decay curve would be heating due to three-body recombination followed by trap loss due to evaporation, or other processes involving secondary collisions [26]. It should be noted that the observed resonances do not resemble the predicted magnetic field dependence for elastic collisions [18]. Therefore, our decay data cannot be explained by elastic collisions leading to evaporation.

We also observed resonant decay at $680 \mathrm{G}$ of a cloud purely in state $|1\rangle$, as shown in Figure 1, b). The fact that this resonance is at the same magnetic field as for the mixture suggests that the observed loss is due to a contamination of the cloud with atoms in state $|2\rangle$. For three-body of decay, our measured $>95 \%$ purity of the preparation of state $|1\rangle$ allows for a maximum of $15 \%$ decay of the cloud, compared to the measured $21 \%$. Further measurements are needed to investigate whether there is an enhancement of losses by secondary collisions, or whether there is a decay mechanism for atoms purely in state $|1\rangle$. 
In conclusion, we observed two decay resonances for the ${ }^{6} \mathrm{Li}$ spin mixture and one resonance in the lowest spin state. Comparing our observations with recent theoretical calculations which exhibit two s-wave Feshbach resonances suggests that the observed decay is a signature of those resonances. Even on resonance, the observed decay happened on a time scale longer than the trap oscillation time, the time for elastic collisions, and the expected sub-millisecond time needed for the formation of Cooper pairs $[27,28]$. Therefore, the ${ }^{6} \mathrm{Li}$ system is well suited for the study of an interacting Fermi gas in the vicinity of an elastic Feshbach resonance, in particular for the search for the phase transition to a superfluid state.

This research was supported by NSF, ONR, ARO, NASA, and the David and Lucile Packard Foundation. C. H. S. acknowledges the support of the Studienstiftung des deutschen Volkes.

Note added: After the submission of this paper several groups reported related results. Measurements of the elastic cross section near the zero crossing associated with the Feshbach resonance have recently been performed by $[19,29]$. Inelastic decay of ${ }^{6} \mathrm{Li}$ fermionic clouds near the Feshbach resonance was recently also observed in the groups of J. E. Thomas [19], and C. Salomon, and for ${ }^{40} \mathrm{~K}$ in the group of D. S. Jin.

[1] S. Inouye, M. R. Andrews, J. Stenger, H.-J. Miesner, D. M. Stamper-Kurn, and W. Ketterle, Nature (London) 392, 151 (1998).

[2] P. Courteille, R. S. Freeland, D. J. Heinzen, F. A. van Abeelen, and B. J. Verhaar, Phys. Rev. Lett. 81, 69 (1998).

[3] A. J. Kerman, V. Vuletić, C. Chin, and S. Chu, Phys. Rev. Lett. 82, 1406 (1999).

[4] S. L. Cornish, N. R. Claussen, J. L. Roberts, E. A. Cornell, and C. E. Wieman, Phys. Rev. Lett. 85, 1795 (2000).

[5] A. J. Leggett, J. Phys. (Paris) 41, C7 (1980).

[6] M. Houbiers, R. Ferwerda, H. T. C. Stoof, W. I. McAlexander, C. A. Sackett, and R. G. Hulet, Phys. Rev. A 56, 4864 (1997).

[7] E. Timmermans, K. Furuya, P. W. Milonni, and A. K. Kerman, Phys. Lett. A 285, 228 (2001).

[8] M. J. Holland, S. J. J. M. F. Kokkelmans, M. L. Chiofalo, and R. Walser, Phys. Rev. Lett. 87, 120406 (2001).

[9] Y. Ohashi and A. Griffin, Rev. Lett. 89, 130402 (2002).

[10] J. N. Milstein, S. J. J. M. F. Kokkelmans, and M. J. Holland, Phys. Rev. A 66, 043604 (2002).

[11] B. DeMarco, S. B. Papp, and D. S. Jin, Phys. Rev. Lett. 86, 5409 (2001).

[12] A. G. Truscott, K. E. Strecker, W. I. McAlexander, G. B. Partridge, and R. G. Hulet, Science 291, 2570 (2001).
[13] F. Schreck, L. Khaykovich, K. L. Corwin, G. Ferrari, T. Bourdel, J. Cubizolles, and C. Salomon, Phys. Rev. Lett. 87, 080403 (2001).

[14] S. R. Granade, M. E. Gehm, K. M. O'Hara, and J. E. Thomas, Phys. Rev. Lett. 88, 120405 (2002).

[15] Z. Hadzibabic, C. A. Stan, K. Dieckmann, S. Gupta, M. W. Zwierlein, A. Görlitz, and W. Ketterle, Phys. Rev. Lett. 88, 160401 (2002).

[16] G. Roati, F. Riboli, G. Modungo, and M. Inguscio, Phys. Rev. Lett. 89, 150403 (2002).

[17] T. Loftus, C. A. Regal, C. Ticknor, J. L. Bohn, and D. S. Jin, Phys. Rev. Lett. 88, 173201 (2002).

[18] M. Houbiers, H. T. C. Stoof, W. I. McAlexander, and R. G. Hulet, Phys. Rev. A 57, R1497 (1998). See also ref. [23].

[19] K. M. O'Hara, S. L. Hemmer, S. R. Granade, M. E. Gehm, J. E. Thomas, V. Venturi, E. Tiesinga, and C. J. Williams, cond-mat/0207717 (2002).

[20] J. Stenger, S. Inouye, M. R. Andrews, H.-J. Miesner, D. M. Stamper-Kurn, and W. Ketterle, Phys. Rev. Lett. 82, 2422 (1999).

[21] J. L. Roberts, N. R. Claussen, S. L. Cornish, and C. E. Wieman, Phys. Rev. Lett. 85, 728 (2000).

[22] C. Chin, V. Vuletić, A. J. Kerman, and S. Chu, Phys. Rev. Lett. 85, 2717 (2000).

[23] M.-O. Mewes, M. R. Andrews, N. J. van Druten, D. M. Kurn, D. S. Durfee, and W. Ketterle, Phys. Rev. Lett. 77, 416 (1996).

[24] H. T. C. Stoof, J. M. V. A. Koelman, and B. J. Verhaar, Phys. Rev. B 38, 4688 (1988).

[25] Private communication with S. J. J. M. F. Kokkelmans and E. Tiesinga.

[26] Private communication with D. Petrov.

[27] M. Houbiers and H. T. C. Stoof, Phys. Rev. A 59, 1556 (1999).

[28] E. Timmermans, Phys. Rev. Lett. 87, 240403 (2001).

[29] M. B. S. Jochim, G. Hendl, J. H. Denschlag, R. Grimm, A. Mosk, and M. Weidemüller, cond-mat/0207098 (2002). 